\title{
A Brain Area for Visual Numerals
}

\author{
Jennifer Shum, ${ }^{1,2}$ Dora Hermes, ${ }^{1,2}$ Brett L. Foster, ${ }^{1,2}$ Mohammad Dastjerdi, ${ }^{1,2}$ Vinitha Rangarajan, ${ }^{1,2}$ \\ Jonathan Winawer, ${ }^{1,3}$ Kai J. Miller, ${ }^{1,2}$ and Josef Parvizi ${ }^{1,2}$ \\ ${ }^{1}$ Stanford Human Intracranial Cognitive Electrophysiology Program (SHICEP), ${ }^{2}$ Laboratory of Behavioral \& Cognitive Neurology, Department of Neurology \\ \& Neurological Sciences, and ${ }^{3}$ Department of Psychology, Stanford University, Stanford, California, 94305
}

Is there a distinct area within the human visual system that has a preferential response to numerals, as there is for faces, words, or scenes? We addressed this question using intracranial electrophysiological recordings and observed a significantly higher response in the high-frequency broadband range (high $\gamma, 65-150 \mathrm{~Hz}$ ) to visually presented numerals, compared with morphologically similar (i.e., letters and false fonts) or semantically and phonologically similar stimuli (i.e., number words and non-number words). Anatomically, this preferential response was consistently localized in the inferior temporal gyrus and anterior to the temporo-occipital incisure. This region lies within or close to the fMRI signal-dropout zone produced by the nearby auditory canal and venous sinus artifacts, an observation that may account for negative findings in previous fMRI studies of preferential response to numerals. Because visual numerals are culturally dependent symbols that are only learned through education, our novel finding of anatomically localized preferential response to such symbols provides a new example of acquired category-specific responses in the human visual system.

\section{Introduction}

A current model of numerical cognition predicts that, within the ventral stream of the human visual system, there is likely to be a region that responds more to visual numerals than to other stimuli (Dehaene, 2011). This hypothesized region for visual numerals should be anatomically consistent across subjects and should respond more to numerals than morphologically, semantically, or phonologically similar stimuli.

Investigations using $\mathrm{fMRI}$, electrophysiological recordings, and electrical stimulation methods have suggested that numerals may be visually processed differently than other stimuli, but these studies have not consistently identified a common brain region within the ventral visual stream that is uniquely responsive to real compared with false (i.e., scrambled) numerals. For example, a recent meta-analysis of the fMRI literature reported variations in the location of activation in the ventral occipitotemporal (VOT) region in studies contrasting numerical stimuli to other kinds of visual stimuli (Arsalidou and Taylor, 2011). Although several fMRI studies did not observe a significant increase of activity in the VOT region in response to visually presented numerals compared with letters (Chochon et al., 1999; Stanescu-Cosson et al.,

Received Sept. 25, 2012; revised Jan. 29, 2013; accepted Feb. 6, 2013.

Author contributions: J.S., B.L.F., M.D., and J.P. designed research; J.S., B.L.F., M.D., V.R., and J.P. performed research; J.S., D.H., B.L.F., M.D., J.W., K.J.M., and J.P. analyzed data; J.S., and J.P. wrote the paper.

This work was supported in part by the National Institutes of Health Grant 1R01 NS0783961 to J.P., Stanford NeuroVentures Program to J.P., National Institutes of Health Grant K99-EY022116 to J.W., and the Medical Scholars Research Program from Stanford University School of Medicine to J.S. We thank the patients for their participation in these studies, Stanford Human Intracranial Cognitive Electrophysiology Program collaborators for insightful comments throughout the study, and staff at Stanford Comprehensive Epilepsy Center for technical assistance during recordings.

The authors declare no competing financial interests.

Correspondence should be addressed to Dr. Josef Parvizi, Laboratory of Behavioral and Cognitive Neurology, Department of Neurology and Neurological Sciences, 300 Pasteur Drive, Stanford, CA 94305. E-mail: jparvizi@stanford.edu.

DOI:10.1523/JNEUROSCI.4558-12.2013

Copyright $\odot 2013$ the authors $\quad 0270-6474 / 13 / 336709-07 \$ 15.00 / 0$
2000; Libertus et al., 2009; Cantlon et al., 2011), one recent fMRI study noted activation in the right, but not left, lateral occipital region for numerals compared with letters, although the same region also displayed a significantly higher response to false (i.e., scrambled) compared with real numerals (Park et al., 2012). This finding is consistent with a previous fMRI study that compared real numerals with scrambled numerals and did not find specific activation in the VOT region (Price and Ansari, 2011). These findings do not rule out the presence of a VOT region with a preferential response to real numerals because clinical evidence suggests that lesions within the ventral temporal region may cause dyslexia for words but not for numbers (Dejerine, 1892; Starrfelt and Behrmann, 2011) and electrical stimulation of the VOT can impair the reading of numerals differently than the reading of alphabetic scripts (Roux et al., 2008). Furthermore, invasive electrophysiological recordings from human subjects have reported event-related potentials in response to numerals in the human VOT (Allison et al., 1999). However, these responses were not compared with important controls, such as morphologically and semantically similar stimuli, which, as noted above, is essential for establishing the selectivity of the response to numerals.

In summary, it is reasonable to conclude that the extant evidence remains inconclusive as to whether there is a consistent anatomical location that has a preferential response to real numerals compared with not only visual stimuli with similar morphological features but also with words that have the same semantic and phonetic features (e.g., number words). In the current study, we designed two experiments to precisely address these questions.

\section{Materials and Methods}

Subjects

This study involved seven ( 3 female and 4 male) right-handed individuals (Table 1) with electrode coverage over the inferior temporal and occipital regions. Electrode placement in these regions was based on clinical data suggesting either posterior temporal or occipital spikes on 
Table 1. Subject demographics

\begin{tabular}{|c|c|c|c|c|c|c|c|}
\hline Subjects & Age (years) & Gender & Education & IQ & Handedness & Seizure focus & Electrode coverage \\
\hline 1 & 48 & Female & College & 69 & R & Right medial temporal lobe & Right temporal lobe \\
\hline 2 & 24 & Male & High school & 65 & $\mathrm{R}$ & Right frontoparietal cortex & Right frontoparietal and temporal lobe \\
\hline 3 & 41 & Male & College & 129 & $\mathrm{R}$ & Right fusiform gyrus & Right temporal and occipital lobe \\
\hline 4 & 46 & Male & High school & 106 & $\mathrm{R}$ & Right calcarine cortex & Right temporal and occipital lobe \\
\hline 5 & 24 & Female & College & 101 & $\mathrm{R}$ & Right medial temporal lobe & Right temporal lobe \\
\hline 6 & 30 & Male & Special education & 56 & $\mathrm{R}$ & Left medial temporal lobe & Left medial temporal lobe \\
\hline 7 & 36 & Female & High school & - & $\mathrm{R}$ & Left medial parietal lobe & Left medial and lateral parietal and temporal lobe \\
\hline
\end{tabular}

scalp EEG or visual auras. Only subject 3 had a seizure focus in the inferior temporal cortex ( $\mathrm{Ta}-$ ble 1). All patients gave their informed consent to participate as subjects in two experiments, and the Stanford University Institutional Review Board approved the study. Five of the subjects were implanted with right and two were implanted with left hemisphere coverage (for subject information, see Materials and Methods and Table 1). None of the subjects had bilateral coverage.

\section{Electrodes}

We used strip and grid electrodes made by AdTech Medical Instrument with the following parameters: $2.3 \mathrm{~mm}$ diameter of exposed recording area $\left(4.15 \mathrm{~mm}^{2}\right)$ and interelectrode distance of 5-10 $\mathrm{mm}$ between the centers of two adjacent electrodes. In total, 1052 electrodes were implanted, 381 of which were located in the VOT region and free of any artifactual or pathological activity. Of these, 157 had a specifically identifiable anatomical location within the visual areas of the VOT region (Fig. 1). Data from these 157 electrodes were used in our analysis.

\section{Electrode localization}

After surgical implantation of the electrodes, postoperative head CT images were aligned to preoperative structural MRIs for localization and visualization of electrode locations with an accuracy of $\pm 5 \mathrm{~mm}$ (median, $2.6 \mathrm{~mm}$ ) as described previously (Hermes et al., 2010). Using single-subject MRIs viewed orthogonally, we identified the neuroanatomical location of each electrode using the occipitotemporal sulcus (OTS), temporo-occipital incisure (toi), and the collateral sulcus $(\mathrm{CoS})$ as anatomical landmarks (Fig. 1).

\section{Electrophysiological recording and analysis}

We recorded signals at $3052 \mathrm{~Hz}$ through a 128-channel recording system made by Tucker Davis Technologies (http://www.tdt.com). We removed channels that had shown any epileptic activity, as determined by the patient's neurologist (J.P.). Off-line, data were notch filtered to remove line noise and rereferenced to the common average.

\section{Electrophysiological experiments}

Two experiments were administered in this study using psychtoolbox (http://psychtoolbox.org/) running on Mac OSX (Fig. 1). The laptop was placed $\sim 70 \mathrm{~cm}$ from the subject's eyes at chest level. Screen resolution was $1280 \times 800$. The height of the stimuli presented on the laptop screen subtended $\sim 2$ degree visual angle.

Experiment 1. Stimuli were adapted from the task used in a previous fMRI study (Price and Ansari, 2011). Subjects were presented with single numerals, letters, scrambled numerals, scrambled letters, or foreign number symbols (Fig. 1).

The scrambled numerals and letters were created from a corresponding numeral or letter stimulus, with the number of pixels, angles, and curves kept as similar as possible while still ensuring that the stimulus
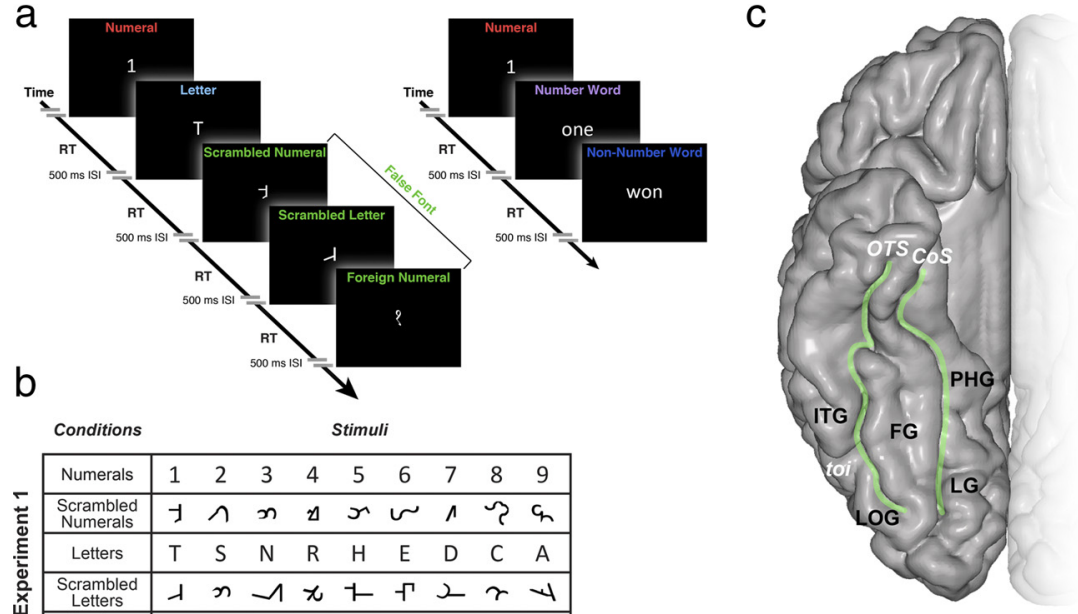

Figure 1. Experimental design and the anatomy of VOT region. $\boldsymbol{a}$, Experiment 1 contains five different conditions: single jects were instructed to respond with a keypad button press whether they could read (button 1) or not read the symbols (button 2). Experiment 2 contains three different conditions: numerals, number words, and non-number words that sound phonetically $\boldsymbol{b}$, Conditions and stimuli for each experiment. $\boldsymbol{c}$, Anatomy of the VOT region and its subregions: ITG, LOG, FG, and PHG can be identified in the individual subjects using anatomical boundaries of $0 T S$, $C O S$, and toi. These regions are illustrated on an MNI brain.

remained unrecognizable. We presented foreign number symbols that were taken from the Devanagari, Thai, and Tibetian languages. We used these stimuli because they are orthographically different from both false fonts and real numerals but yet represent numerals in different cultures. The stimuli were presented in white font (Calibri, size 100) on a black background. The subjects were instructed to respond with a keypad button press whether they could read the symbol on the screen. The experiment was self-paced with the trial length being determined by the subject's response time. The interstimulus interval was $500 \mathrm{~ms}$. Each stimulus was repeated four times and presented randomly (180 stimuli total).

Experiment 2. Subjects were shown three different categories of stimuli: a numeral, a number word, or a non-number word that sounded phonetically similar to a numeral (Fig. 1). Each numeral had a corresponding stimulus in the other two categories (Fig. 1). The stimuli were presented in white font (Times, size 90) on a black background. The subjects were instructed to read the stimulus aloud with trial duration being determined by the subjects' response time. The interstimulus interval was $500 \mathrm{~ms}$. In total, 20 trials of numerals (10 single numeral and 10 double digit numerals), 20 trials of corresponding number words, and 20 trials of corresponding non-number words were presented in random order to each patient. According to analyses of the Contemporary American English Corpus (425 million words, 1990-2011; http://corpus.byu. 
edu/coca/) and Google Books American English (155 billion words, 1810-2009; http://googlebooks.byu.edu/), there was no difference in usage frequency of the number words and non-number words used in this study.

\section{Defining response and baseline}

Given that the aim of our study was to identify response selectivity within proximal anatomical regions, we needed to use the most functionally and spatially precise electrophysiological signal recorded with our subdural electrodes. For this reason, we used the high-frequency broadband (HFB, also known as high $\gamma$ ) as our measure for the induced local brain activity. Because of the surface area of our electrodes (i.e., $\sim 4.15 \mathrm{~mm}^{2}$ ), we estimate that each recording site registers signals from a pool of $\sim 0.5$ million neurons (Pakkenberg and Gundersen, 1997). For this reason, we consider changes in power across the HFB range to reflect local neuronal population activity. Furthermore, HFB activity provides a regioninvariant measure of local activation and correlates best with population firing rates and fMRI BOLD responses (Logothetis et al., 2001; Manning et al., 2009; Miller et al., 2010; Miller, 2010; Flinker et al., 2011; Hermes et al., 2011; Ray and Maunsell, 2011; Parvizi et al., 2012). Naturally, we are mindful that the profile of the recorded electrophysiological activity is very rich and one could potentially analyze responses in the slower bands of activity (e.g., $\alpha$ or $\theta$ band). However, the choice of HFB as the main measure of local brain activity was directly motivated by the central hypothesis and the specific aim of the study. Unlike the HFB activity, changes in lower frequency narrow-band oscillations are less consistently correlated with population firing rates and fMRI BOLD responses, and more importantly, show a wide spatial distribution and variability on the cortical surface (Crone et al., 1998; Voytek et al., 2010; Foster and Parvizi, 2012).

To quantify HFB power changes within each electrode, we first bandpass filtered the data between 65 and $150 \mathrm{~Hz}$ using a zero-phase lag finite impulse response filter (eegfilt; EEGLAB; http://sccn.ucsd.edu/eeglab/) and then applied a Hilbert transform to obtain the instantaneous amplitude. We then calculated the power of this time series and normalized it by subtracting and dividing each time point by the SD of the baseline. For the baseline, we chose the mean of the power in the $200 \mathrm{~ms}$ before stimulus onset for all stimuli from all conditions. During this baseline interval, subjects were shown a blank screen with a black background. We then averaged the normalized power of HFB activity for all stimuli within the same condition by aligning the trials with respect to the stimulus onset as described in detail in our previous studies (Dastjerdi et al., 2011; Foster and Parvizi, 2012).

\section{Statistical analysis}

Responses for each trial were defined as the mean normalized HFB power calculated from 200 to $500 \mathrm{~ms}$ after stimulus onset. This $200-500 \mathrm{~ms}$ window was chosen because the average response onset and offset across all conditions in inferior temporal electrodes was 239 and $514 \mathrm{~ms}$, respectively. Onset and offset times were determined as described in our previous publication (Dastjerdi et al., 2011). To identify electrodes that preferentially respond to numerals, we performed a two-sided $t$ test for each electrode located in either the inferior temporal gyrus (ITG), the lateral occipital gyrus (LOG), the fusiform gyrus (FG), or the parahippocampal gyrus (PHG), comparing responses to numerals versus letters and false fonts (Experiment 1), and numerals versus number words and non-number words (Experiment 2). The degree of preferential response to numerals for each electrode was then defined using the $t$ values calculated by the two-sided $t$ test. An electrode was considered to have preferentiality for numerals if there was significantly larger HFB response during numerals compared with the other conditions in both Experiment 1 and Experiment 2. All $t$ tests were corrected for multiple comparisons using an false discovery rate correction for all tested electrodes.

\section{fMRI experiment}

Six healthy volunteers were recruited for fMRI data acquisition to measure the extent of signal dropout. Data were acquired on a 3T GE scanner for an independent experiment (600-900 volumes per subject, matrix size $64 \times 64, \mathrm{TR}=2 \mathrm{~s}, \mathrm{TE}=29 \mathrm{~ms}$, flip angle $=77^{\circ}$, voxel size $2.5 \mathrm{~mm}$ isometric). All functional images were motion corrected, and field maps were acquired in separate scans using a spiral sequence to correct for image distortion. The mean signal was calculated for each subject and normalized to a range of $0-1$ for each subject, and then averaged across the six subjects.

\section{Results}

At the group level, a large mantle of the VOT region encompassing its key anatomical regions of interest were covered, although specific electrode positioning differed between subjects. Before analyzing the recorded electrophysiological responses, electrodes containing artifacts or epileptic activity were excluded. As explained above, we used event-related power changes in the HFB range (i.e., $65-150 \mathrm{~Hz}$, also known as high $\gamma$ ) as the measure of regional activation because, unlike slower oscillatory signals or event-related potentials, the HFB serves as the most functionally and spatially precise signal.

In all subjects, electrophysiological signals were recorded while subjects performed two experiments. In Experiment 1 (Fig. 1 ), we tested the hypothesis that there is an area within the inferior temporal region that has a significantly larger response to numerals compared with morphologically similar stimuli, such as letters or false fonts (i.e., scrambled numerals, scrambled letters, or foreign numerals). In Experiment 2 (Fig. 1), we tested the hypothesis that the preferential response to numerals remains larger even if compared with words with similar semantic and/or phonetic content (e.g., "1" compared with "one" or "won"; "6" compared with "six" or "sex," etc.). A repeated-measures ANOVA with a Greenhouse-Geisser correction determined that mean response times were not significantly different among conditions in Experiment $1\left(F_{(1.009,6.052)}=1.332, p=0.293\right)$ or $\mathrm{Ex}^{-}$ periment $2\left(F_{(1.050,6.298)}=2.380, p=0.172\right)$.

To explore the anatomical coordinates of the preferential response to numerals, we estimated the degree of preferential response to numerals for each electrode across all subjects by contrasting the extent to which responses to numerals differed from morphologically similar stimuli (false font and letters) and from semantically and phonetically similar stimuli (number words and non-number words) using a $t$ test (see Materials and Methods). Taking the $t$ values from each electrode, we visualized the resulting spatial distribution of preferential response across the VOT region for each experiment (Fig. 2). Strikingly, the measure of numeral preferentiality across subjects displayed consistent maxima within ITG for numerals compared with both morphologically similar and semantically/phonetically similar stimuli (Fig. 2). The location of this area was consistently anterior to the toi. The MNI coordinates of the peak of the smoothed comparison between numerals and morphologically similar stimuli in the right hemisphere were $51,-54$, and -24 .

To localize the significant responses to visual numerals in each individual brain, we performed a two-sided $t$ test for each electrode located in the VOT. We compared responses to numerals versus letters and false fonts (Experiment 1 ) and numerals versus number words and non-number words (Experiment 2) for all electrodes. An electrode was considered to have a preferential response to numerals if there was significantly larger activity during numerals compared with the other conditions in both Experiment 1 and Experiment 2 ( $p<0.05$, false discovery rate corrected for all 157 VOT electrodes). Seven such electrodes were identified among four subjects (Figs. 3 and 4, red circles with black border). The electrodes with preferential response to numerals showed a remarkably consistent location: 6 of 7 numeral preferential electrodes were located on the ITG, and the other 
numeral preferential electrode was located on the border of the ITG and FG (Fig. 3).

We surveyed the activity patterns in all electrodes across subjects and experiments and found an interesting blueprint of response characteristics (Fig. 4). Although neuronal populations in the ITG had significant preferentiality for numerals compared with morphologically similar stimuli and phonologically and semantically similar stimuli (Fig. 4, red circles), their neighboring sites showed preferentiality to numerals compared with number words or non-number words (Experiment 2, Fig. 4, red diamonds) but not to morphologically similar stimuli, such as false fonts (Experiment 1, Fig. 4, red stars). Indeed, many sites adjacent to the ITG (especially the ones located in the LOG) showed significantly higher response to false fonts compared with real numerals (Fig. 4, green circles). By contrast, sites on the medial bank of the OTS (where the visual word form area is usually localized) had preferentiality for numerals compared with morphologically similar stimuli (Fig. 4, red diamonds) but failed to show preferentiality to numerals compared with number words or nonnumber words (Fig. 4, red stars).

Although we had recordings from the right as well as the left hemisphere and responses to numerals were seen in the same ITG location (Fig. 2), we could not explore in detail the issue of lateralization because our coverage in the left hemisphere was not as extensive as in the right hemisphere, and none of the subjects was implanted with electrodes bilaterally.

Our finding of a strong and preferential electrophysiological response to numerals in the ITG raised the question of why its preferential response to numerals compared with morphologically similar stimuli has not been reported in the fMRI literature. Given the proximity of the ITG to the auditory canal and transverse sinus, we hypothesized that the air-bone inter-

face within the petrous bone of the inner ear in conjunction with the venous flow of the transverse sinus (Winawer et al., 2010) causes a significant fMRI signal dropout (i.e., an eclipse zone), which in turn affects the quality of fMRI signals acquired from this area. To test this hypothesis, we recruited six healthy subjects in whom we measured the scope of the fMRI eclipse zone. We choose healthy control subjects, rather than the epilepsy patients who had participated in our electrophysiological experiments, to avoid factors, such as craniotomy and partial brain resection after epilepsy surgery, which could confound the extent of fMRI signal dropout. As seen in Figure 5, we were able to confirm the presence of the fMRI eclipse zone in the ITG region within the healthy control subjects. The ITG region with a preferential response to numerals identified in our subjects falls surprisingly close to, or within, the fMRI eclipse zone.
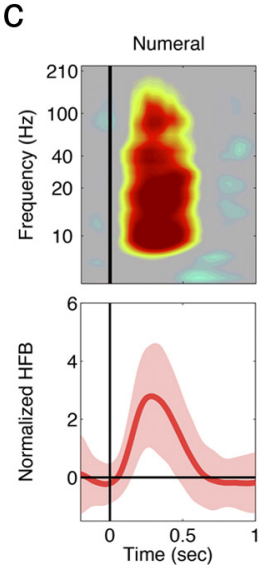
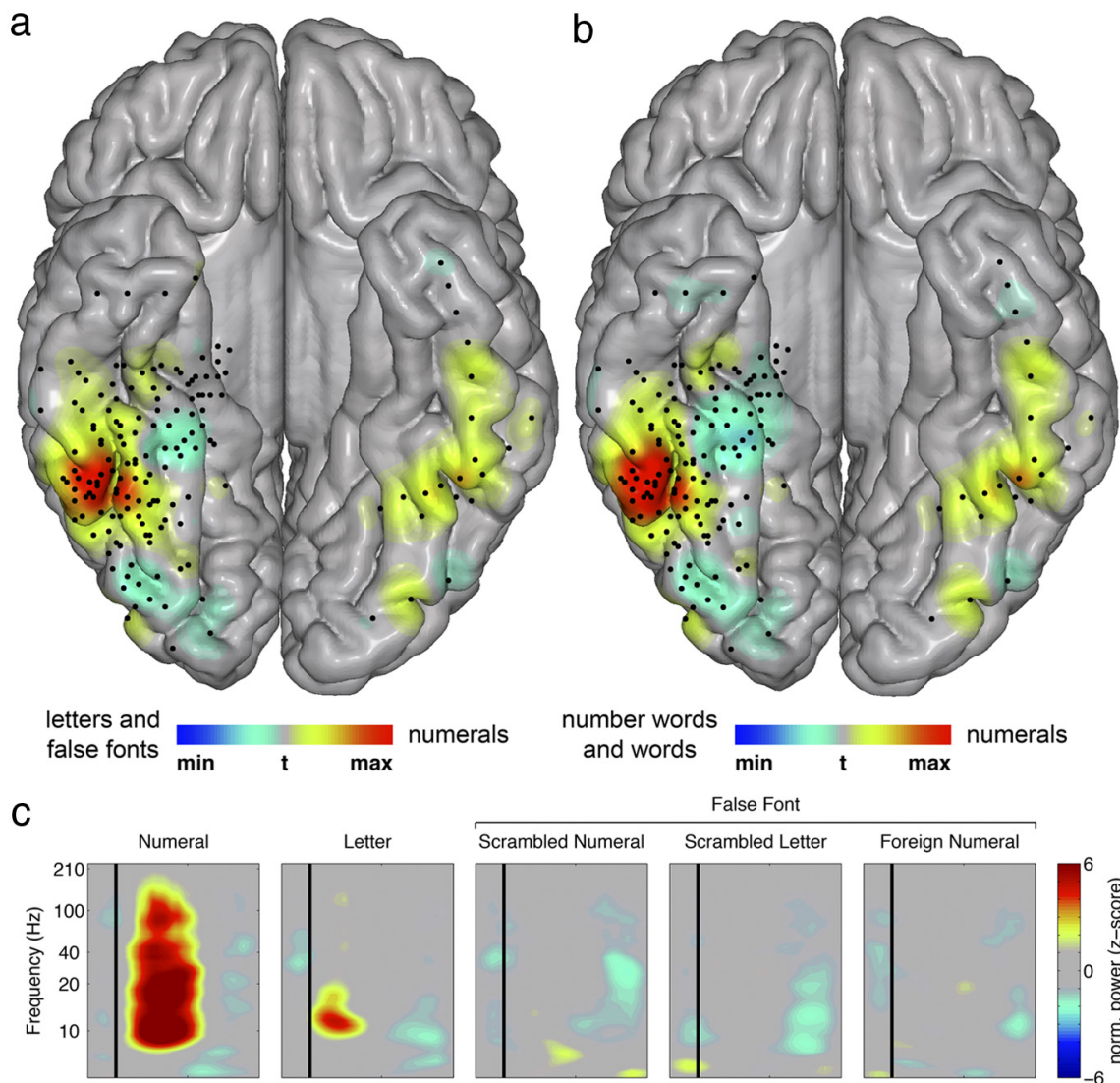

False Font
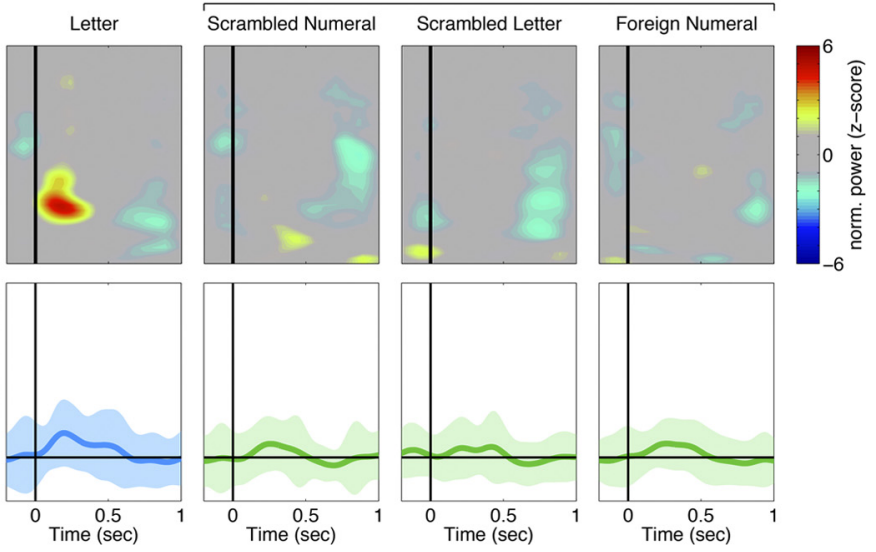

Preferential response to numerals is in the high-frequency broadband range and located within the ITG. $\boldsymbol{a}, \boldsymbol{b}$, For all Experiment $1(\boldsymbol{a})$ and Experiment $2(\boldsymbol{b})$. The smoothed distribution of the $t$ values shows a preferential response to numerals in the ITG. The peak of the smoothed comparison between numerals and morphologically similar stimuli in the right hemisphere (ExperThere were a total of 157 electrodes. c, Activity in the ITG during Experiment 1 in an individual subject (Subject 2). Top, Timeuency analysis during the five different visual stimuli from Experiment 1. The $y$-axis represents the log scale of frequencies from HFB power traces over time during the five different visual stimuli (mean \pm SD shown as solid \pm shaded area). Stimulus onset is at 0 . There is a greater HFB response during the processing of numerals than other conditions.

\section{Discussion}

Using intracranial EEG, we have identified the location of a neuronal population within the ITG with greater activation to visual symbols denoting numerals compared with morphologically, semantically, or phonologically similar symbols. The novelty of our finding is both in identifying the precise anatomical location of neurons with a preferential response to visual numerals as well as in highlighting that these neurons are imbedded within a larger pool of neurons that respond nonpreferentially to visual symbols that have lines, angles, and curves. Further study of this cortical area is essential to understand developmental and cultural factors that explain how a certain population of neurons within a pool of nonpreferential neurons gains preferential responses to visual symbols, such as numerals, which are only learned throughout education 
a

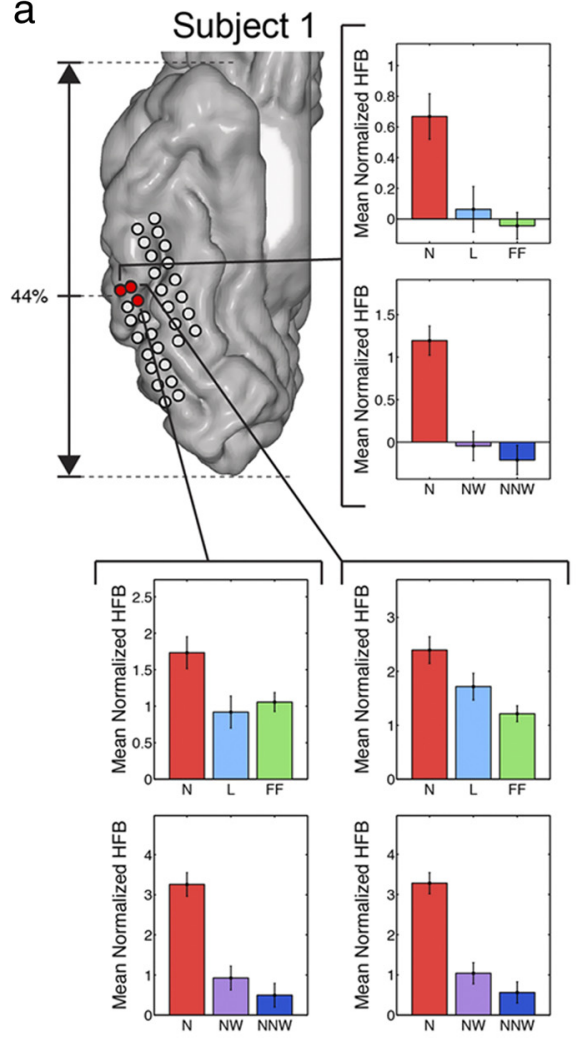

b

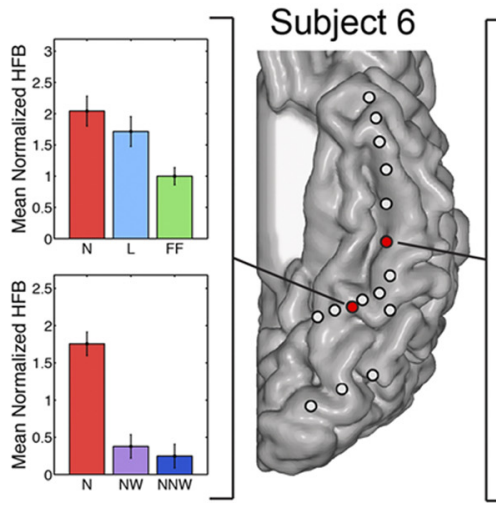

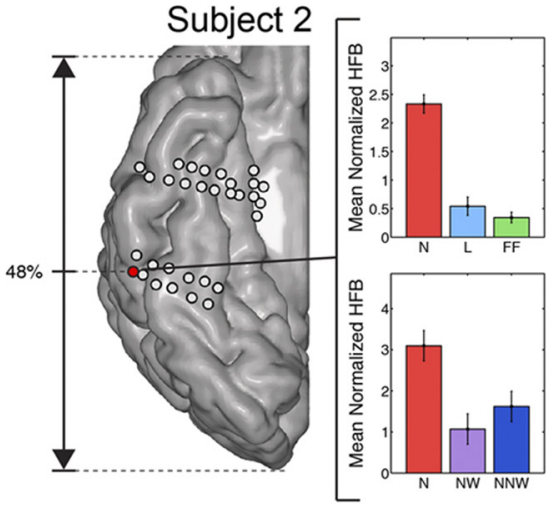
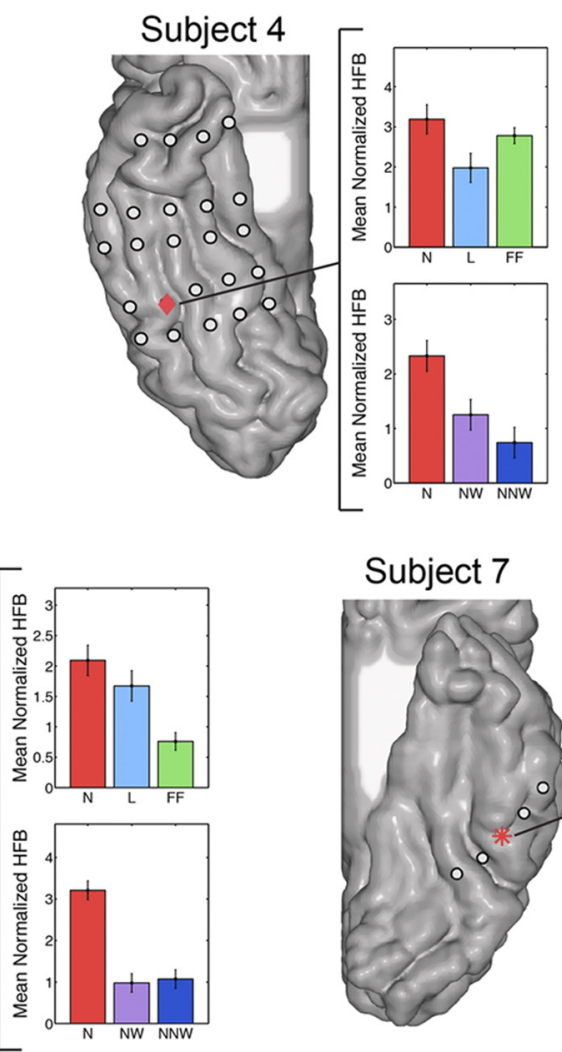
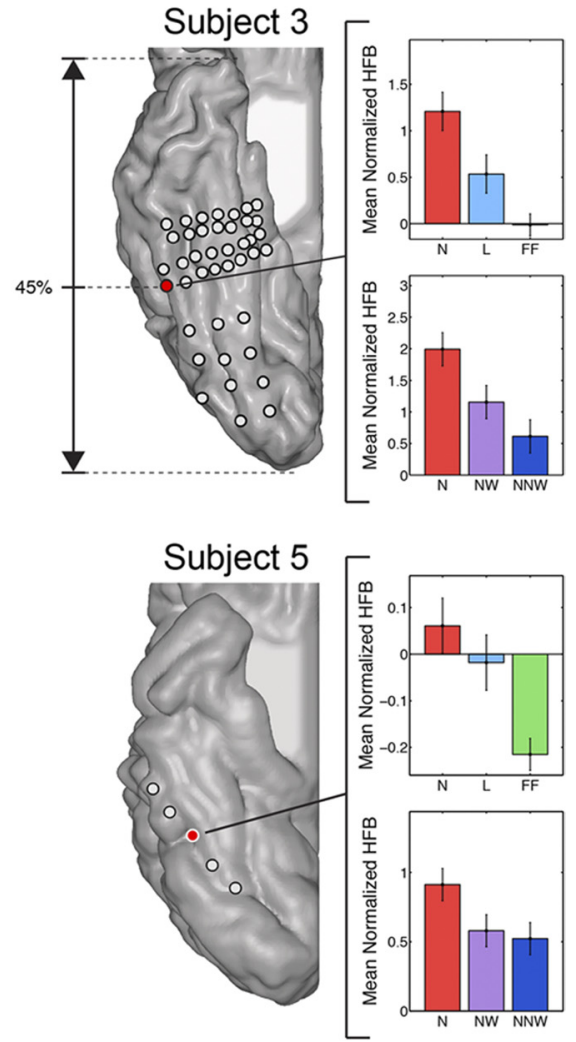

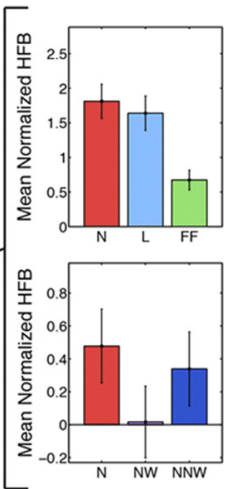

$\square$ Numeral

Letter

False Font

Number Word

Non-Number Word

Figure 3. Individual profile of responses to numerals and control stimuli in the ITG. The MRI reconstruction of the inferior surface of the right ( $\boldsymbol{a}$ ) and left ( $\boldsymbol{b}$ ) hemispheres shows the location and pattern of electrode coverage in each individual patient. Different coverage pattern across patients is the result of different clinical needs of individual patients. Electrodes with significant preferential response to numerals are shown in red with a black border. Neuronal populations under these electrodes had a significantly larger response to numerals compared with other stimuli in both Experiment 1 and Experiment 2. Red electrodes with a white border represent electrodes that showed a numeral preferential trend (i.e., a larger response to numerals was statistically significant in one experiment but showed a trend in the other). Star electrodes showed a significantly larger response to numerals compared with other conditions in only Experiment 1 . Diamond electrodes had a significantly larger response to numerals in only Experiment 2. Bar plots represent the mean normalized HFB power \pm the SE for each condition in the selected electrode for Experiment 1 (top) and Experiment 2 (bottom). There is consistent anatomical location of the most preferential response in the first three subjects with denser coverage with the percentage distance from the occipital to the temporal pole indicated.

(Dehaene and Cohen, 2007; Ansari, 2008). Our finding of the precise anatomical coordinates of this preferential response can be explored further in human subjects from other cultures where visual numerals are symbolized differently.

We emphasize that we have used the term "preferential" rather than "selective" to highlight the fact that electrodes with the maximal response to numerals also responded (although much less strongly) to other symbols containing lines, curves, and angles. Moreover, some regions without a clear preferential response to numerals were also activated above baseline during processing of numerals, even though their response to numerals was significantly weaker than their response to con- trol stimuli (for discussions about category-specific representations see, e.g., Cohen and Tong, 2001; Kanwisher, 2010; DiCarlo et al., 2012; Mur et al., 2012). To determine the exact nature of numeral processing in the ITG, future studies need to explore the structural and functional connections between the ITG and its adjacent VOT structures, earlier visual cortex, and parietofrontal areas involved in language and memory.

The precise anatomical localization of neuronal populations with a preferential response to visual numerals is important for future studies of their relationship with other brain areas that are involved in higher aspects of numerical cognition and the semantic processing of numbers. As discussed in 


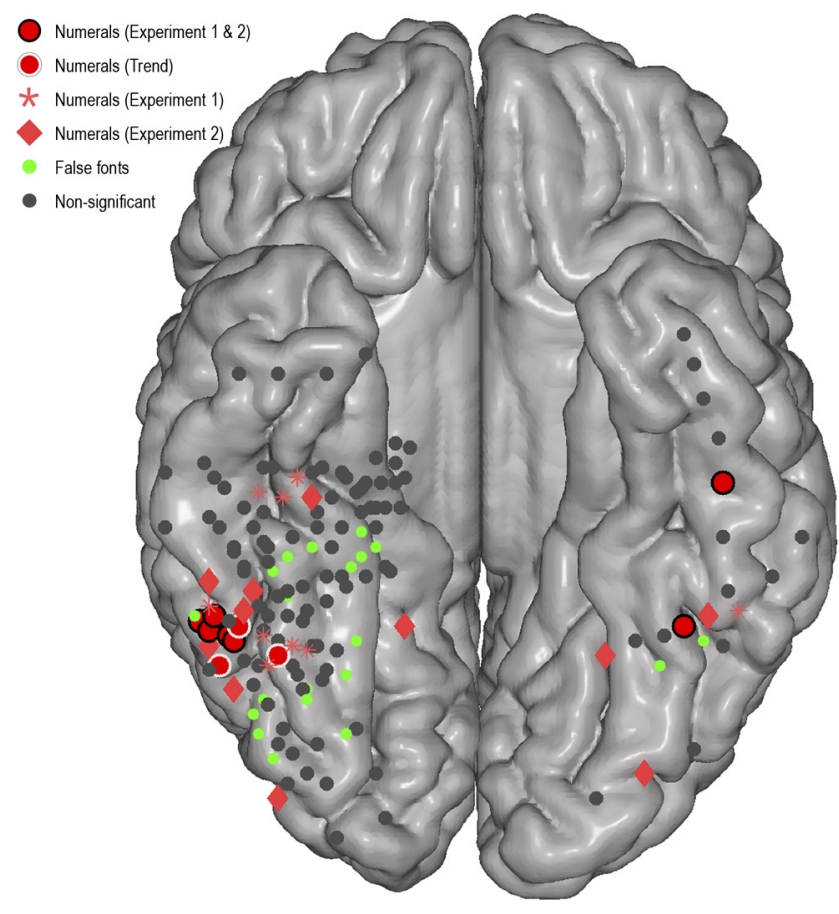

Figure 4. Location of preferential responses to numerals and false fonts in all subjects depicted on normalized brain. Electrode coverage ( $n=157)$ over the four anatomical regions (ITG, FG, LOG, and PHG) in all seven subjects is displayed on a normalized MNI brain. Red circles with black or white borders or stars and diamonds represent regions with varying degrees of preferential response to numerals (for definitions, see Fig. 3). Green electrodes represent electrodes with significantly larger response to false fonts than to numerals. This figure shows two main findings: (1) electrodes with most preferential response to numerals (red with black border) are located in the ITG; and (2) sites adjacent to the ITG have a stronger response to morphologically similar false fonts than real numerals (green electrodes).
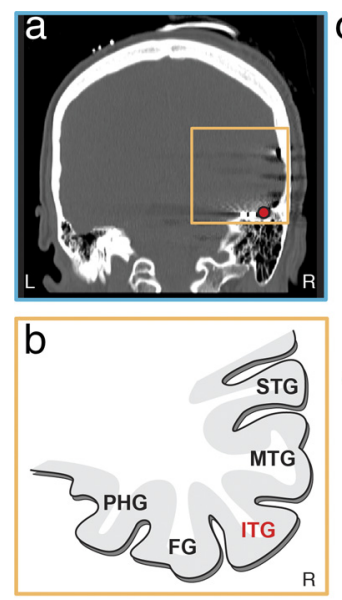

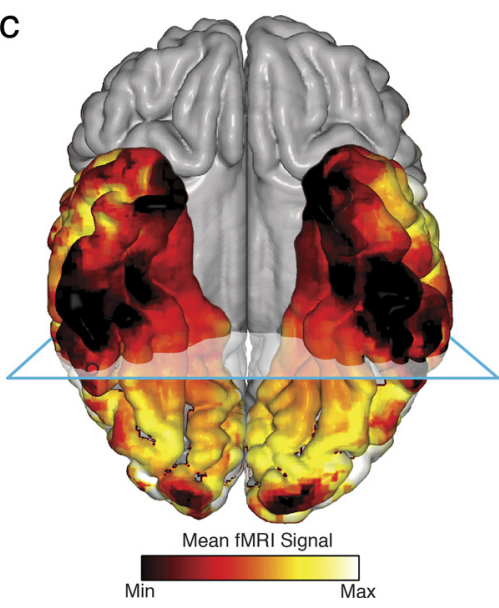

Figure 5. fMRI eclipse zone. $\boldsymbol{a}, \mathrm{CT}$ image of a subject's brain in the coronal plane showing a strip of electrodes under the temporal lobe. The electrode with preferential response to numerals is marked with a red circle. There is air-filled petrous bone located underneath the electrode. $\boldsymbol{b}$, Schematic of the gyri surrounding the ITG. MTG, Medial temporal gyrus; STG, superior temporal gyrus. $c$, The mean fMRI signal in 6 healthy control subjects was normalized to a range of 0-1 for each individual and then averaged across subjects and rendered on a standard MNI brain. The area with a preferential response to numerals indicated by a red circle falls nearby the core of the signal dropout. c, Blue plane represents the coronal plane for $\boldsymbol{a}$ and $\boldsymbol{b}$.

the literature of numerical cognition (e.g., Ansari, 2008; Dehaene, 2011), any neuronal population involved in visual processing of numbers must be located in a region of the brain that has anatomical access to not only the visual cortices but also to the parts of the brain that are involved in auditory and written language, arithmetic, and memory functions. The ITG is precisely a region with such connections, and therefore, is best suited to be pruned by education and experience for its preferential function in the visual encoding of numerals. Conventional anatomical tracing studies in nonhuman primates have shown that the posterior ITG region around the medial and lateral banks of the OTS has strong connections with cortices of peristriate, lateral prefrontal, superior temporal sulcus, medial temporal, temporoparieto-occipital, and intraparietal sulcus regions (Kuypers et al., 1965; Pandya and Kuypers, 1969; Martin-Elkins and Horel, 1992). It is likely that a similar structural and functional network also exists in the human brain. Future studies will determine the bidirectional connectivity of the human ITG and the lateral parietal cortical sites, such as the intraparietal sulcus and angular gyrus, which are known to be involved in numerical cognitive functions in humans and nonhuman primates and whose dysfunction is associated with dyscalculia (Ansari, 2008; Nieder and Dehaene, 2009; Butterworth, 2010; Dehaene, 2011). What remains to be determined is the exact nature of such bidirectional information processing within the network ranging from the time visual symbols are detected and the semantic representations of numerical quantities are retrieved to the time when arithmetic calculations are performed successfully. Given the high temporal resolution and precise anatomical information of electrocorticography signals, we think that future studies with simultaneous recordings across regions of the brain will elucidate the complex spatiotemporal dynamics of the brain networks involved in numerical cognition.

It is noteworthy that the anatomical location of the preferential response to numerals is close, but not identical, to the area within the OTS that has preferential response to words (i.e., the so-called visual word form area) (Nobre et al., 1994). This anatomical proximity is important because the two sites are frequently coengaged in visual processing of words and numbers that co-occur frequently in our everyday life and because both neuronal populations need to use the same anatomical pathways to connect with other brain regions engaged in, for example, language function. However, the fact that numerals and words use different population of neurons is particularly informative because there are morphological similarities between letters and numerals, and yet, many patients with severe deficits in reading words and letters, such as Dejerine's patient Monsieur C, have been described to have much lesser, if any, deficits in their ability to read numerals (for a comprehensive review, see Starrfelt and Behrmann, 2011).

The location of the preferential response to numerals within the fMRI eclipse zone can explain the previous negative fMRI findings of preferential response to numerals compared with false fonts and suggests that it will be relatively more difficult to measure a reliable BOLD signal in this area. Because the fMRI signal dropout depends on the individual anatomy and the scanning parameters, it should be possible to record data from these regions in certain individuals and using better scanning parameters in terms of voxel size and slice orientation to increase the signal quality (Weiner and GrillSpector, 2012). Nonetheless, a nontrivial epistemological impact of our finding is that our knowledge about a brain region might be limited or even obscured with one method but be highlighted by another. 


\section{References}

Allison T, Puce A, Spencer DD, McCarthy G (1999) Electrophysiological studies of human face perception: I. Potentials generated in occipitotemporal cortex by face and non-face stimuli. Cereb Cortex 9:415-430. CrossRef Medline

Ansari D (2008) Effects of development and enculturation on number representation in the brain. Nat Rev Neurosci 9:278-291. CrossRef Medline

Arsalidou M, Taylor MJ (2011) Is $2+2=4$ ? Meta-analyses of brain areas needed for numbers and calculations. Neuroimage 54:2382-2393. CrossRef Medline

Butterworth B (2010) Foundational numerical capacities and the origins of dyscalculia. Trends Cogn Sci 14:534-541. CrossRef Medline

Cantlon JF, Pinel P, Dehaene S, Pelphrey KA (2011) Cortical representations of symbols, objects, and faces are pruned back during early childhood. Cereb Cortex 21:191-199. CrossRef Medline

Chochon F, Cohen L, van de Moortele PF, Dehaene S (1999) Differential contributions of the left and right inferior parietal lobules to number processing. J Cogn Neurosci 11:617-630. CrossRef Medline

Cohen JD, Tong F (2001) Neuroscience: the face of controversy. Science 293:2405-2407. CrossRef Medline

Crone NE, Miglioretti DL, Gordon B, Lesser RP (1998) Functional mapping of human sensorimotor cortex with electrocorticographic spectral analysis: II. Event-related synchronization in the $\gamma$ band. Brain 121:2301-2315. CrossRef Medline

Dastjerdi M, Foster BL, Nasrullah S, Rauschecker AM, Dougherty RF, Townsend JD, Chang C, Greicius MD, Menon V, Kennedy DP, Parvizi J (2011) Differential electrophysiological response during rest, selfreferential, and non-self-referential tasks in human posteromedial cortex. Proc Natl Acad Sci U S A 108:3023-3028. CrossRef Medline

Dehaene S (2011) The number sense: how the mind creates mathematics, Ed 2. Oxford: Oxford UP.

Dehaene S, Cohen L (2007) Cultural recycling of cortical maps. Neuron 56:384-398. CrossRef Medline

Dejerine JM (1892) Contribution a l'etude anatomo-pathologique et clinique des difrentes varietes de cite' verbale. C R Seanc Soc Biol 4:61-90.

DiCarlo JJ, Zoccolan D, Rust NC (2012) How does the brain solve visual object recognition? Neuron 73:415-434. CrossRef Medline

Flinker A, Chang EF, Barbaro NM, Berger MS, Knight RT (2011) Subcentimeter language organization in the human temporal lobe. Brain Lang 117:103-109. CrossRef Medline

Foster BL, Parvizi J (2012) Resting oscillations and cross-frequency coupling in the human posteromedial cortex. Neuroimage 60:384-391. CrossRef Medline

Hermes D, Miller KJ, Noordmans HJ, Vansteensel MJ, Ramsey NF (2010) Automated electrocorticographic electrode localization on individually rendered brain surfaces. J Neurosci Methods 185:293-298. CrossRef Medline

Hermes D, Miller KJ, Vansteensel MJ, Aarnoutse EJ, Leijten FS, Ramsey NF (2011) Neurophysiologic correlates of fMRI in human motor cortex. Hum Brain Mapp 33:1689-1699. CrossRef Medline

Kanwisher N (2010) Functional specificity in the human brain: a window into the functional architecture of the mind [Inaugural article]. Proc Natl Acad Sci U S A 107:11163-11170. CrossRef Medline

Kuypers HG, Szwarcbart MK, Mishkin M, Rosvold HE (1965) Occipitotemporal corticocortical connections in the rhesus monkey. Exp Neurol 11: 245-262. CrossRef Medline

Libertus ME, Brannon EM, Pelphrey KA (2009) Developmental changes in category-specific brain responses to numbers and letters in a working memory task. Neuroimage 44:1404-1414. CrossRef Medline

Logothetis NK, Pauls J, Augath M, Trinath T, Oeltermann A (2001) Neuro- physiological investigation of the basis of the fMRI signal. Nature 412: 150-157. CrossRef Medline

Manning JR, Jacobs J, Fried I, Kahana MJ (2009) Broadband shifts in local field potential power spectra are correlated with single-neuron spiking in humans. J Neurosci 29:13613-13620. CrossRef Medline

Martin-Elkins CL, Horel JA (1992) Cortical afferents to behaviorally defined regions of the inferior temporal and parahippocampal gyri as demonstrated by WGA-HRP. J Comp Neurol 321:177-192. CrossRef Medline

Miller KJ (2010) Broadband spectral change: evidence for a macroscale correlate of population firing rate? J Neurosci 30:6477-6479. CrossRef Medline

Miller KJ, Hermes D, Honey CJ, Sharma M, Rao RP, den Nijs M, Fetz EE, Sejnowski TJ, Hebb AO, Ojemann JG, Makeig S, Leuthardt EC (2010) Dynamic modulation of local population activity by rhythm phase in human occipital cortex during a visual search task. Front Hum Neurosci 4:197. CrossRef Medline

Mur M, Ruff DA, Bodurka J, De Weerd P, Bandettini PA, Kriegeskorte N (2012) Categorical, yet graded, single-image activation profiles of human category-selective cortical regions. J Neurosci 32:8649-8662. CrossRef Medline

Nieder A, Dehaene S (2009) Representation of number in the brain. Annu Rev Neurosci 32:185-208. CrossRef Medline

Nobre AC, Allison T, McCarthy G (1994) Word recognition in the human inferior temporal lobe. Nature 372:260-263. CrossRef Medline

Pakkenberg B, Gundersen HJ (1997) Neocortical neuron number in humans: effect of sex and age. J Comp Neurol 384:312-320. CrossRef Medline

Pandya DN, Kuypers HG (1969) Cortico-cortical connections in the rhesus monkey. Brain Res 13:13-36. CrossRef Medline

Park J, Hebrank A, Polk TA, Park DC (2012) Neural dissociation of number from letter recognition and its relationship to parietal numerical processing. J Cogn Neurosci 24:39-50. CrossRef Medline

Parvizi J, Jacques C, Foster BL, Withoft N, Rangarajan V, Weiner KS, GrillSpector K (2012) Electrical stimulation of human fusiform faceselective regions distorts face perception. J Neurosci 32:14915-14920. CrossRef Medline

Price GR, Ansari D (2011) Symbol processing in the left angular gyrus: evidence from passive perception of digits. Neuroimage 57:1205-1211. CrossRef Medline

Ray S, Maunsell JH (2011) Different origins of $\gamma$ rhythm and high- $\gamma$ activity in macaque visual cortex. PLoS Biol 9:e1000610. CrossRef Medline

Roux FE, Lubrano V, Lauwers-Cances V, Giussani C, Démonet JF (2008) Cortical areas involved in Arabic number reading. Neurology 70:210-217. CrossRef Medline

Stanescu-Cosson R, Pinel P, van De Moortele PF, Le Bihan D, Cohen L, Dehaene S (2000) Understanding dissociations in dyscalculia: a brain imaging study of the impact of number size on the cerebral networks for exact and approximate calculation. Brain 123:2240-2255. CrossRef Medline

Starrfelt R, Behrmann M (2011) Number reading in pure alexia: a review. Neuropsychologia 49:2283-2298. CrossRef Medline

Voytek B, Canolty RT, Shestyuk A, Crone NE, Parvizi J, Knight RT (2010) Shifts in $\gamma$ phase-amplitude coupling frequency from $\theta$ to $\alpha$ over posterior cortex during visual tasks. Front Hum Neurosci 4:191. CrossRef Medline

Weiner KS, Grill-Spector K (2012) The improbable simplicity of the fusiform face area. Trends Cogn Sci 16:251-254. CrossRef Medline

Winawer J, Horiguchi H, Sayres RA, Amano K, Wandell BA (2010) Mapping hV4 and ventral occipital cortex: the venous eclipse. J Vis 10(5):1. CrossRef Medline 